\title{
Ragione e smarrimento. Verga, Pirandello, Sciascia ${ }^{1}$
}

\author{
Vincenzo Consolo
}

\begin{abstract}
$\grave{E}$ un itinerario attraverso i tre grandi scrittori siciliani, tre modi diversi di contemplare il male e l'infelicità che attanagliano il mondo e la storia. Mentre Verga assume un atteggiamento di rassegnazione, Pirandello, logico e laico, vuole invece capire le «ragioni per cui la sentenza è stata pronunciata». Sciascia va più in là e cerca di individuarne i responsabili.

Parole chiave: arcaico, lo «strappo nel cielo di carta», la stasi della storia, caruso, patto sociale.

\section{Abstract}

A tour of the work of the three greatest Sicilian writers, three different modes of contemplating the evil and unhappiness that afflict the world throughout history. While Verga attains an attitude of resignation, the logical, secular Pirandello, attempts to understand "the reasons behind the pronunciation of such a sentence». Sciascia is more concerned with the search for individuation and responsibility.
\end{abstract}

Key words: archaic, the "tear in the paper sky», the stasis of history, caruso, social contract.

La marea ha investito Acitrezza, la casa del nespolo dei Toscano, la famigliola di «buona e brava gente di mare» soprannominata Malavoglia. Discesa, la marea niente ha lasciato più come prima. Ora quel cerchio chiuso e immobile di vita umile, ma che si credeva armoniosa, s'è spezzato per colpa del nipote di padron 'Ntoni, per quella vaga bramosia dell' ignoto che ha fatto infrangere al giovane i comandamenti della religione del paese-nido, del focolare domestico. Ora il fato impassibile e beffardo, che si rivela nella lotta per la vita, ai livelli infimi di un villaggio chiuso fra il mare e la sciara, assoggettata a una natura avara, torva, rovinosa, a una storia lontana, incompresa, ora il fato s'è accanito contro i Malavoglia. E sono morti, perdite, esclusioni, rinunzie, follie, diso- 
nori. 'Ntoni lascia il paese e sconta il peccato col carcere, l'esilio volontario. Maruzza, madre ammantata, immobile avanti al mare, ai marosi, priva di lacrime, lamento, parola, privata di figli, marito, si porta le mani ai capelli, urla nera nel cuore, muore di pena. La giovane Lia fugge per perdersi, vendersi nei vicoli infami della città. L'altra, la Mena, la sant'Agata che stava sempre al telaio, rinunzia all'amore, al matrimonio con Alfio, si rinserra nella casa prigione - con un controrito mesto rimette alla treccia la spadina d'argento che le era stata tolta a suo tempo per spartire i capelli, disporla alle nozze. La barca, la Provvidenza, resa inservibile dal naufragio, viene venduta. La casa del nespolo è ceduta ai creditori e abbandonata: vuota, è ora, al pari di un tempio, dissacrata dall'invasione di estranei, di infedeli che con indifferenza spazzano via, disperdono le reliquie superstiti. Il patriarca, scacciato dalla casa-tempio, si pietrifica per il dolore, perde vigore e ragione: il suo salmodiare si fa sconnesso, i suoi proverbi sono ormai senza capo né coda. Muore. Questo vinto dal fato, muore solo, ignorato in un tetro stanzone all'albergo dei poveri.

Acitrezza, la Trezza, l'intreccio. Forse nessun romanzo moderno è privo d'intreccio - v'è una ripetizione ossessiva di sciagure come per spietato gioco del caso - nessuna narrazione è così priva di romanzesco come I Malavoglia. Un poema narrativo, un'epica popolana, un'odissea chiusa, circolare, che dà il senso, nelle formule lessicali, nelle forme sintattiche, nel timbro monocorde, nel tono salmodiante, nei proverbi gravi e immutabili come sentenze giuridiche o versetti di sacre scritture, Bibbia, Talmud o Corano, dà il senso della mancanza di movimento, dell'assenza di sviluppo, suggerisce l'immagine della fissità: della predestinazione, della condanna. Parte da Trezza il romanzo verghiano, il ciclo dei Vinti, parte dal luogo estremo, limen, soglia di passaggio in cui l'antica lava scivola e si scioglie nel mare, nell'incertezza assoluta della natura. Parte dall'oscura grotta, dal dolore senza nome, dall'urlo della Locca, la demente, che vaga per le strade e invoca, reclama il figlio morto, scomparso in fondo al mare.

Un villaggio di poche case, Trezza, con la piazza, la chiesa, l'osteria, la sciara, le barche amarrate sopra la spiaggia; il mare che s'infrange e spumeggia contro i faraglioni; e l'Etna alle spalle, nero e fumante, in eterna minaccia. Un luogo di pura esistenza, di mero accadimento. Il luogo della treccia, del nodo mai sciolto della tragedia.

Fuggono i superstiti della tempesta, s'allontanano dal mare, migrano oltre il Simeto, il biviere di Lentini, in un paese di storia, memoria, intricato come una medina, con palazzi, case, piazze, chiese, conventi, con vaste terre intorno, con tante chiuse. Nella consistenza della terra, vivono ancora come naufraghi sulla zattera dove può accadere ogni crudeltà, scatenarsi ogni ferocia. Più soli che ad Acitrezza, qui a Vizzini hanno sostituito alla religione della tradizione, degli affetti, il fanatismo della roba, del possesso. E non c'è scampo per gli eretici né per i fedeli: la mancanza della roba pone ai margini, alla mercé della violenza; il suo accumulo, quanto più si fa ossessivo, tanto più nutre il tumore della sofferenza, come ha scritto Lawrence, porta alla desolazione, alla morte. I Malavoglia si chiude in un'alba in cui, dopo l'addio di 'Ntoni alla casa del 
nespolo, ai vivi e ai morti, l'addio al paese, a poco a poco il mare si sbianca, le stelle, i Tre Re, la Puddara, impallidiscono, le casipole riemergono a una a una dal buio della notte: l'infinito tempo scorre ancora indifferente, riprende la vicenda sempre uguale, la mesta melodia del poema, la lingua trepida, contratta, la sapienza fallace del dire ereditato.

Mastro-don Gesualdo si apre in un'alba ancora buia, arrossata dai bagliori del fuoco che divampa in un palazzo di nobili decaduti, abitato da due larve d'uomo, don Diego e don Ferdinando Trao, fissati in una loro inderogabile alterigia, in una loro folle ri-vendicazione quali discendenti di un antico, libertino re di Spagna: Virtutem a sanguine traho è il loro motto. Ma è, il loro, un sangue che si è ormai devitalizzato, si è quasi spento. Nel palazzo è anche la giovane sorella Bianca, vestale del cadente mausoleo di memorie ormai sbiadite, che nell'alba, assieme alla intimità della casa, viene violata: un uomo fugge dalla sua stanza; il popolaccio, richiamato dal suono delle campane, irrompe nelle vuote sale, schiamazza, tocca, calpesta, strappa brandelli di stoffa alle pareti.

Da questo palazzo al centro del paese e da una piccola, plebea casa contigua, che per l'esca di legna ha rischiato di bruciare anch'essa, inizia il dramma, si svolge l'odissea. Che è scandita questa volta in lingua distesa, in una prosa incivilita: la melodia sommessa del poema è ormai lontana. Un'energia vitale, una primigenia schiettezza, verità, irrompe nel palazzo, vivifica e salva, ma scema e si spegne a sua volta. Gesualdo, il muratore che diventa ricco, sposa Bianca: connubio chimerico che somma solitudine a solitudine, pena a pena, che non darà mai frutto, sollievo. — Le pesche non s'innestano sull'ulivo — dice amaramente l'uomo che ha calpestato i doni dell'umile Deodata, che ha avuto, quale unico frutto e ingannevole conforto, l'accumulo spasmodico dei beni. La duchessa di Leyra, Isabella, la figlia formale ma non reale, dissiperà la roba nel palazzo di Palermo, dove il vecchio muratore morirà, in un'alba livida, nell'indifferenza e nel disprezzo dei servi.

Perché in Verga questa concezione metastorica, questa visione del mondo in cui sono assenti suggestioni populistiche, risentimenti moralistici, polemiche sociali? La sua poetica, la sua ideologia letteraria esclude le ragioni, la ragione della o nella storia, la concezione progressiva della storia, afferma la condanna, la sconfitta ineludibile dell'uomo. Eschilo e Leopardi dirigono il suo sguardo, lo portano alla tragedia senza catarsi, alla matrigna natura in cui sola consolazione è il profumo della «lenta» ginestra. Sappiamo delle due stagioni letterarie di Verga, dei primi suoi romanzi cosiddetti mondani culminati con la Storia di una capinera, di una fanciulla malmonacata che finisce nella follia e nella morte. Sappiamo della sua crisi, della sua conversione, della sua rivoluzione tematica e stilistica. Avviene a Milano questa sua caduta da cavallo, nella città in cui si era trasferito nel 1872, nella Milano erede del laico illuminismo dei Verri e del Beccaria e della cristiana provvidenzialità del Manzoni. Avviene in una città in quegli anni, in preda alla prima rivoluzione industriale, invasa dalla fede nelle «magnifiche sorti e progressive», presa nella lotta del «Progresso contro il Regresso", come recitava la didascalia del Ballo Excelsior, rappresentato 
alla Scala in occasione dell'Esposizione Nazionale del 1881. Verga, spaesato, si ritrae davanti a tanto attivismo ottimistico, a tanta modernità. Ad aggravare la sua crisi sono anche le notizie che giungono in quegli anni dalla Sicilia, dalle inchieste parlamentari e da quella di Sonnino e Franchetti, sulle condizioni dei contadini, degli zolfatari, dei bambini che lavorano in miniera, sul brigantaggio e la mafia. Una realtà che lo scrittore sino a quel momento aveva eluso, una memoria che aveva sepolto dentro di sé. "A questo punto", dice il Sapegno, "nasce in lui il bisogno di risalire alle origini e suscitare le memorie pure della sua infanzia e riprender contatto con la sua terra. Alla quale egli tornava con l'animo del figliol prodigo, come all'unico bene che ancora gli rimanesse, intatto e solido dopo tanta dissipazione». C'era stato dunque nello scrittore il rifiuto del capitalismo attivistico, delle ragioni progressive degli imprenditori e degli ingegneri milanesi e l'assunzione, nella nostalgia, nel recupero delle memorie pure dell'infanzia, l'assunzione dell'arcaico, immobile capitalismo rurale siciliano: arcaismo e immobilità che bandivano ragione e storia, conducevano alla metastoria, alla concezione negativa della condizione umana. Fioriva la poesia, la ginestra su questo terreno desolato, in una lingua nuova, un italiano di primo grado, irradiato di dialettalità.

Nella hall il portiere consegnò un biglietto a Pirandello, che subito lo lesse, si scusò con Niccodemi e si diresse spedito verso il salone. Là trovò Verga, in compagnia del barone Zappalà Asmundo. Il vecchio puntellò le mani sul bastone facendo le mosse di volersi alzare, ma Pirandello, svelto, lo fece riaccomodare sulla poltrona. Gli si sedette di fronte. I due si guardarono negli occhi, non osarono profferir parola.

-Quando riparte, maestro? - chiese il barone per rompere il silenzio.

—Domani $[\ldots]$ - sussurrò Pirandello, e continuò a guardare quel vecchio in marsina nera, la testa che s'ergeva dal solino inamidato, i folti capelli bianchi, il viso asciutto, il naso diritto, i baffi arricciati, lo sguardo severo, profondo. Gli sembrò, Verga, la personificazione di don Ippolito Laurentano de $I$ vecchi e i giovani, il principe che non accetta il nuovo tempo, che si richiude a Colimbètra, nell'orgoglio, nella follia. Gli sembrò superbo e ostinato come il padre suo. Comparò sé, più giovane di quasi trent'anni, il suo viso con i segni incisi del tormento, il cranio nudo, la barbetta precocemente incanutita, gli occhi sgomenti per il guardare oltre il teatro, oltre l'apparenza, comparò la sua con la ferma maschera, quasi pietrificata del nobile vegliardo.

- Perdonami Luigi - disse finalmente Verga. - A te tutta la mia gratitudine, ma dall'Italia ufficiale non voglio niente!

Pirandello abbassò il capo, si sentì in qualche modo complice dell'ufficialità. Si scosse, tirò fuori dalla tasca dei fogli, glieli porse.

— Ecco - disse, - ecco il discorso [...] L'ha ricopiato per voi mia figlia, Lietta $[\ldots]$

— Ringraziamela tanto [...] Ti seguo, Luigi, leggo quanto scrivi [...] Ho visto a Milano quella tua commedia [...] Come si chiama? Cosi è [...]

- se vi pare - completò Pirandello.

- Quel personaggio, la velata [...] Che pena!

Ripiombarono di nuovo nel silenzio. 
Verga inforcò gli occhiali, spiegò i fogli e si mise a leggere. Pirandello lo osservò ancora e gli sembrò lontano, irraggiungibile, chiuso in un'epoca remota, irrimediabilmente tramontata. Pensò che al di là dell'esterna ricorrenza, delle formali onoranze, in quel tempo di lacerazioni, di violenza, di menzogna, in quel tramonto, in quella notte della pietà e dell'intelligenza, il paese, il mondo, avrebbe ancora e più ignorato, offeso la verità, la poesia dello scrittore. Pensò che quel presente burrascoso e incerto, sordo alla ritrazione, alla castità della parola, ebbro d'eloquio vano poteva essere rappresentato solo col sorriso desolato, con l'umorismo straziante, con la parola che incalza e che tortura, la rottura delle forme, delle strutture, la frantumazione delle coscienze, con l'angoscioso smarrimento, il naufragio, la perdita dell'io.

Si tolse gli occhiali, il vecchio, piegò i fogli e se li mise in tasca.

— Lo leggerò a casa, stanotte — disse — Dormo ormai così poco [...] Aspetto la morte, a occhi aperti $[\ldots]$-.

Chiese:

- Cosa stai scrivendo, Luigi?

- Ho appena terminato una commedia [...] Sei personaggi in cerca d'autore $[\ldots]$

Mi sono permesso di riportare qui un mio brano narrativo, tratto da L'olivo e l'olivastro, su un episodio reale, storico, vale a dire sull'incontro a Catania, nel 1920, in occasione dei festeggiamenti per l'ottantesimo compleanno dell'autore de I Malavoglia, tra Verga appunto e Pirandello, in cui quest'ultimo era stato incaricato di pronunciare al Teatro Bellini il discorso ufficiale. Incontro tra due grandi scrittori, fra due mondi. Il passaggio, in loro, dall'antica tragedia alla moderna commedia, al dramma. Dramma che viene da drao, fare, agire.

Pirandello rompe quel cerchio chiuso, fatale, quel salmodiare monocorde dei vinti verghiani, quel mondo reale, concreto, quel coro compatto che sulla scena cantava e lamentava la tragedia. Aveva interrotto quel canto, Pirandello, aveva disposto su un piano lineare la prosa, logica, serrata, in una lingua di secondo grado, grammaticale, letteraria regolata. Aveva fatto irrompere nell'antico coro dionisiaco il moderno spirito socratico, quello che Nietzsche individua nel passaggio dalla tragedia eschilea a quella euripidea. Introduce, Pirandello, la riflessione, il ragionamento, la dialettica, la tesi e l'antitesi, il processo verbale, il movimento, l'azione mentale, il dibattito come in una immaginaria aula di un tribunale o una stanza borghese, quella stanza che Giovanni Macchia chiama della «tortura».

Non accetta più la condanna, questo scrittore logico e laico, indaga e vuol capire le ragioni per cui la sentenza è stata pronunciata. Scompone quel gruppo uniforme e compatto, quella moltitudine di mortali, quel plurimo attore che è il coro e guarda al singolo soggetto, all'individuo, alla persona che si fa personaggio. Afferma che nessuna entità esterna, metafisica e oscura, nessun fato ha decretato la condanna, ma che la crisi, il crollo, la catastrofe non è fuori, ma dentro l'individuo, s'è installata dentro di lui sin dal momento in cui ha preso coscienza del suo stare nel mondo, di far parte dell'umanità e di quella umanità dominata da regole e forme che si chiama società. Società dominata 
e dominante che cerca, attraverso la forma, di vanificare, annullare la vita, che scava e crea vuoti profondi dentro l'individuo, che scompone, moltiplica l'unità, impone all'io frantumato, che vuole scansare la follia o la morte, una maschera. La catastrofe non può più essere narrata o rappresentata in forma tragica, ma con una prosa "civile» che nasce da quel sentimento del contrario, da quel reagente "terribile» che si chiama umorismo. Ne Il fu Mattia Pascal Anselmo Paleari informa Adriano Meis, il fu Mattia, che l'Elettra di Sofocle viene rappresentata a Roma in un teatro di marionette.

Or senta un po' - dice il signor Paleari - che bizzarria mi viene in mente! Se, nel momento culminante, proprio quando la marionetta che rappresenta Oreste è per vendicare la morte del padre, sopra Egisto e la madre si facesse uno strappo nel cielo di carta del teatrino, che avverrebbe? Oreste rimarrebbe sconcertato da quel buco nel cielo. [...] Oreste sentirebbe ancora gl'impulsi della vendetta, vorrebbe seguirli con smaniosa passione, ma gli occhi, sul punto, gli andrebbero lì, a quello strappo, donde ora ogni sorta di mali influssi penetrerebbero nella scena, e si sentirebbe cader le braccia. Oreste insomma diverrebbe Amleto. Tutta la differenza, signor Meis, tra la tragedia antica e la tragedia moderna consiste in ciò, creda pure: in quel buco nel cielo di carta.

Ecco, esemplarmente, come Pirandello introduce l'umorismo, quell'umorismo con il quale Amleto fa recitare ai guitti la scena dell'assassinio del padre.

I mali influssi da quello strappo nel cielo di carta arrivano sì sulla scena, ma penetrano il personaggio, lo sconvolgono, lo straziano, lo torturano, lo spingono al dubbio, alla perdita di sé, al limite della follia, alla violenza verso gli altri o verso se stesso. Il cielo di carta è la forma che blocca, pietrifica, uccide la vita. La tragedia, in questo arresto, non è più possibile, s'è già consumata, essa non può che essere evocata, tentata, reclamata da fantasmi, da informi creature della fantasia che cercano l'autore per avere corpo e anima, avere sul palcoscenico riconoscimento e ragione.

Riflessione, ragionamento, discorso logico, serrato, spasmodica ricerca d'una verità inesistente, abbiamo detto. Ma c'è alla radice di tutto questo, alla sorgente di ogni filosofismo e di ogni sofisma, una frattura, uno jato, un anello che non tiene. C'è il dubbio sulla realtà, sulla sua apparenza, c'è la messa in mora di essa, il suo abbandono; c'è il trasferimento dello sguardo e della mente sull'incerto piano del relativismo. Dove tutto e niente è possibile, è infinita fuga nella ricerca di un centro che è ovunque e da nessuna parte; il prezzo è l'arresto d'ogni impulso, istinto, scissione del corpo dallo spirito, ritrazione, segregazione, solitudine, pietrificazione, annullamento. Un'umanità, questa dell'universo pirandelliano, di ansia e tremore in un antinferno privo di giudizio e di destinazione. Personaggi, i suoi, dall'io diviso, schizoidi, folli savi che arrivano fino alla soglia della ribellione, del trapasso nel freddo e nel buio della volontà e della ragione. Non c'è quasi nessun vero folle nel vastissimo e complesso mondo pirandelliano, non c'è in esso dispiegata una fenomenologia di psicotici. Sarebbe caduto, lo scrittore, in questo caso, nel vecchio naturalismo zoliano. Da cui si è lucidamente staccato, da cui è infinitamente lontano. Il 
suo mondo, scosso sì, agitato da una vena di follia, è un sistema di giudizio, è un universo di poesia. Enrico $I V$, che recita la sua pazzia, è diventato pazzo battendo la testa per una caduta da cavallo. E qui allora non siamo più, ci sembra, nel campo della psichiatria, ma semmai in quello della neurologia (siamo in ogni caso, in una improbabilità clinica).

Grande profeta, con Kafka, Musil, Proust, Joyce, Svevo, Eliot, Pirandello ha rappresentato la condizione nostra di uomini moderni. E il suo nucleo poetico, la sua metafora, come in una reazione a catena, s'allarga e s'invera quanto più il mondo nostro scientificizzato, tecnologizzato, priva l'uomo di certezze, lo spinge nell'inquietudine, nello smarrimento, nell'angoscia.

Cultura, sì, letteratura, trattati filosofici e scientifici, come Les altérations de la personalité di Alfred Binet, avevano contribuito alla concezione del suo mondo poetico. Ma le vere, profonde radici di quel mondo affondano nella terra di Sicilia. La storia personale di Pirandello, la sua famiglia, la sua infanzia, Agrigento e le persone, i personaggi di quella società ci spiegano in modo esemplare la nascita, in quel luogo estremo d'Europa, in quell'Isola, in quella città dell'arresto del tempo, della stasi della storia, la nascita di un così geniale interprete della crisi della civiltà nostra occidentale. Pochi fatti vogliamo sottolineare di questa sua storia personale: il ricordo, nella primissima infanzia, di un'eclisse di sole visto dal Caos in tempo di epidemia, di colera; il conflitto profondo e lacerante col padre Stefano e l'attaccamento alla madre; l'esperienza della zolfara, di quel giallo inferno di desolazione, di fatica disumana, di continuo rischio di morte; ultimo, e sigillo d'una pena, di un'antica paura di oltrepassare la soglia, di scivolare nella follia, la follia oggettiva, come trasferita, della moglie Antonietta. Gramsci per primo aveva individuato, al di là di ogni filosofia, di definizione teorica del tempestoso mondo di Pirandello, le profonde radici siciliane della sua poetica.

Sulla scorta di Gramsci, è Leonardo Sciascia che chiama il mondo pirandelliano "pirandellismo di natura», Sciascia che non considera l'infelicità individuale e sociale come dato dell'esistenza o come metafora della crisi di una civiltà, ma come portato della storia, e della storia in un ben preciso contesto, in un'Isola, la Sicilia, in una città, Agrigento, nel suo desolato territorio di chiarchiàri, di ammassi pietrosi e di miniere di zolfo, in un paese che gli Arabi chiamarono Rahal-maut, villaggio morto.

Qui, a Racalmuto è nato Sciascia, qui è vissuto fino alla giovinezza, in questo paese eminentemente pirandelliano, sotto la spessa ombra, nella notte illune sopra il caos, il disperato irrazionalismo pirandelliano. Da questo re di Tebe, da questo padre annientatore 1'Edipo scrittore ha dovuto liberarsi. È Viktor Sklovskij del resto ad affermare che quella letteraria è una storia continua di parricidi. In più punti della sua opera, in saggi, racconti, Sciascia dice di questo suo processo di liberazione, della ricerca di sé in un'altra realtà, in un altrove, in un ideale luogo di chiara ragione, in una agognata società di giustizia e di libertà.

«C'è stata una stagione della nostra vita in cui Pirandello diede nome a tutto il nostro sgomento, ai nostri umani rapporti, alla nostra pietà. E riflettendo 
scopriamo che il nostro incontro con Pirandello è avvenuto, nel tempo e nello spazio, ad un pericolosissimo incrocio: negli anni dell'adolescenza, e in una piccola città della Sicilia. Scendemmo allora nell'opera pirandelliana come in un averno di specchi e d'ombre: e ne traemmo disperate rivelazioni, allucinazioni logiche - fino all'ossessione», scriveva Sciascia già nel ' 54 . E aggiungeva, mutuando un verso di Cardarelli, che poi «avrebbe bevuto in ben altre cantine».

Risale dunque lo scrittore dall'Averno pirandelliano per approdare nella Toscana dell'armonia rinascimentale, fra gli eredi «rondisti» della società della cultura e della lingua. E, per la Toscana, per il «toscanizzato» Manzoni, giunge nella patria della Ragione, fra gli illuminati fondatori della moderna nostra civiltà. L'Averno pirandelliano era sì per Sciascia quello esistenziale di specchi e di ombre, di allucinazioni logiche, di ossessioni, ma era anche, nella realtà, nella dura realtà della storia e della contingenza, il pozzo profondo, il labirinto infernale di gallerie e cunicoli della zolfara. Zolfara che i due scrittori hanno guardato in modo diverso, opposto. Pirandello dall'alto, da figlio di un proprietario di zolfara, di una famiglia in continua paura e angoscia per le incerte fortune di quella impresa su cui poggiava il loro stato, la loro identità sociale. «Il nucleo familiare è l'oscuro germe da cui nascono gli infiniti casi pirandelliani», afferma Macchia.

Sciascia guarda la miniera, di zolfo dal basso, da dentro anzi, da figlio e nipote di zolfatari.

Uomini del mio sangue furono carusi nelle zolfare, picconieri, braccianti nelle campagne. [...] Ad un momento, ecco il punto buono, ecco il capomastro, l'impiegato; ed io che non lavoro con le braccia e leggo il mondo attraverso i libri. Ma è tutto troppo fragile, gente del mio sangue può tornare nella miseria $[\ldots]$ Finché l'ingiustizia sarà nel mondo, sempre, per tutti, ci sarà questo nodo di paura.

La paura di precipitare nel fondo della zolfara non crea più smarrimento, angoscia esistenziale, sofistica disgregazione, arresto e perdita di ragione e verità, ma fermo recupero di realtà, di storia, della ragione che illumina le tessere sconnesse della storia, di una scrittura, di una letteratura che fosse luce, ordine e carità, anelito, in quel mondo di ingiustizia e di violenza, a una umana compagine di ragione ed equità. Sciascia trasferisce il dibattito dal chiuso torturante della stanza borghese nella pubblica piazza della socialità, della politica, con uno scarto che annulla isolamento e solitudine, vanifica allucinazioni e fantasmi.

Tutta l'opera di Sciascia è una necessaria, essenziale, lucida e serrata (anche se man mano sempre più disperata) conversazione in Sicilia. Una conversazione che tende a realizzare una comunicazione assoluta, una convivenza sociale più giusta, vale a dire più umana. Una convivenza dove nessuno, individuo, Stato o potere d'ogni tipo, politico, giudiziario, religioso o finanziario deve infrangere le regole della convivenza, deve offendere il cittadino. Tutta la poe- 
tica di Sciascia è contenuta nel primo suo libro, Le parrocchie di Regalpetra, lì si trovano i temi che lo scrittore svilupperà negli altri suoi libri. Libri tutti che formano un unico libro sulla Sicilia, racconti che partono quasi sempre da Regalpetra, da Racalmuto. Da qui snodando quel filo ad alta tensione morale che attraversa la Sicilia, l'Italia, tutto il contesto socio-culturale in cui si trova immerso il destinatario della sua narrazione o del suo ragionamento, si trova il suo interlocutore.

A Regalpetra c'è un luogo privilegiato da cui si osserva la realtà sociale, in cui di essa realtà si conversa: il Circolo della Concordia. Il patto concordatario su cui si fonda il Circolo, lo stato democratico, viene violato quando, chi ha la responsabilità della sua gestione (individui, organi o gruppi di potere), agisce contro lo Statuto; da qui i temi sciasciani del potere politico e del potere giudiziario degenerati, i temi di organizzazioni criminali come la mafia che minano lo statuto. E da qui, quando alla degenerazione segue l'omicidio (il massimo dell'infrazione perché viola il primo e sommo principio del patto sociale: il rispetto della vita umana), da qui l'interesse di Sciascia per il romanzo poliziesco, la narrazione di più alto valore civile. Perché l'indagine poliziesca non è che il tentativo di ricucire i fili dello strappo, operato nel tessuto sociale, attraverso l'individuazione e la condanna del colpevole. Ma così non vanno le cose in Sicilia, in Italia, dove l'omicidio non è che l'ultima manifestazione di una serie di delitti che il potere politico degenerato mette in atto attraverso quel suo braccio armato che è la mafia. L'individuazione dell'assassino o degli assassini comporta l'individuazione dei mandanti, comporta l'indagine del potere su se stesso, la messa sotto accusa e la condanna di se stesso. Nei polizieschi di Sciascia dunque gli assassini non sono quasi mai individuati e mai puniti perché si tratta di delitti politici, perché i suoi sono romanzi politici.

Dopo Il Consiglio d'Egitto, in cui metteva in campo i grandi temi illuministici e manzoniani dell'impostura, della tortura e della pena di morte, dopo La morte dellinquisitore in cui narrava degli odiosi misfatti dell'Inquisizione, Sciascia scriveva una serie di racconti polizieschi che vanno da II giorno della civetta, A ciascuno il suo, Il contesto fino a Todo Modo, in cui si è indagata e rappresentata la storia del potere politico italiano di quegli anni. L'affaire Moro è come la dimostrazione e il sigillo reale e tragico di ogni sua invenzione letteraria. Il cavaliere e la morte e Una storia semplice sono gli ultimi due passi d'addio, gli ultimi due racconti di disperata sfiducia nella possibilità di sondare gli oscuri meandri del potere politico-mafioso. Ultime amare dimostrazioni della sconfitta della ragione. L'insondabilità del potere politico, sappiamo, sarà incrinata nella realtà da giudici di nuova cultura, di nuova moralità, che pagheranno con la vita questo loro civile, generoso azzardo. Parlo di Giovanni Falcone e Paolo Borsellino.

Verga, Pirandello, Sciascia: tre scrittori che, ciascuno a suo modo, con la loro poetica, con la loro lingua, denunciano un unico atavico, storico dramma, in Sicilia, nel nostro Paese: l'assenza di una società civile, il trionfo del violento nazionalismo tribale, lo smarrimento per l'assenza della ragione. 